\title{
High-order geodesic coupling of shear-Alfvén and acoustic continua in tokamaks
}

\author{
Paulo Rodrigues ${ }^{1}$ and Francesca Cella ${ }^{1,2}$ \\ ${ }^{1}$ Instituto de Plasmas e Fusão Nuclear, Instituto Superior Técnico, \\ Universidade de Lisboa, 1049-001 Lisboa, Portugal. \\ ${ }^{2}$ Dipartimento di Energia, Politecnico di Milano, Via Ponzio 34/3, 20133 Milan, Italy.
}

(Dated: July 30, 2021)

\begin{abstract}
High-order plasma shaping (mainly elongation and shift, as opposed to low-order toroidicity) is shown, under certain conditions, to open gaps in the coupled shear-Alfvén and acoustic continua at frequencies significantly above the values predicted by previous theories. Global eigenmodes in these gaps, which lie between those of geodesic acoustic modes (GAMs) and toroidicity-induced Alfvén eigenmodes (TAEs), are found unstable to hot-ion populations typical of tokamak operation, whilst their fundamental resonances with circulating particles are shown to take place at velocities near the geometric mean of the Alfvén and sound speeds. Therefore, such eigenmodes are expected to be observed near the predicted frequencies at operating tokamaks, playing a still unexplored role in magnetohydrodynamic spectroscopy as well as in the stability of next-step fusion experiments.
\end{abstract}

\section{INTRODUCTION}

Continuous spectra of the magnetohydrodynamics (MHD) operator are central to a variety of phenomena dominated by inhomogeneous magnetic fields [1-3], from astrophysical plasmas to fusion devices. Their origin lies on vanishing coefficients in the eigenvalue equation

$$
\mathcal{F}(\boldsymbol{\xi})+\mu_{0} \rho \omega^{2} \boldsymbol{\xi}=0
$$

for small plasma displacements $\boldsymbol{\xi} e^{-i \omega t}$, where $\mu_{0}$ is the magnetic constant and $\rho$ is the mass density, with

$$
\begin{aligned}
\mathcal{F}(\boldsymbol{\xi})=(\nabla \times \mathbf{B}) \times[ & \nabla \times(\boldsymbol{\xi} \times \mathbf{B})] \\
+[\nabla \times \nabla & \times(\boldsymbol{\xi} \times \mathbf{B})] \times \mathbf{B} \\
& +\mu_{0} \nabla(\gamma P \nabla \cdot \boldsymbol{\xi}+\boldsymbol{\xi} \cdot \nabla P)
\end{aligned}
$$

the ideal-MHD operator [3, 4], $P$ and $\mathbf{B}$ the equilibrium pressure and magnetic field, while $\gamma=5 / 3$. As a simple but rather conveying example, let $\rho$ be uniform, $\nabla \cdot \boldsymbol{\xi}=0$, and $\mathbf{B}=B(x) \mathbf{e}_{z}$, which turns Eq. (1) into [1]

$$
\frac{d}{d x}\left[\left(\omega^{2}-k_{\|}^{2} v_{\mathrm{A}}^{2}\right) \frac{d}{d x} \tilde{\xi}_{x}\right]-k^{2}\left(\omega^{2}-k_{\|}^{2} v_{\mathrm{A}}^{2}\right) \tilde{\xi}_{x}=0,
$$

where $\boldsymbol{\xi}=\tilde{\boldsymbol{\xi}}(x) \exp \left[i\left(k_{y} y+k_{z} z\right)\right], k_{\|}=\mathbf{k} \cdot \mathbf{b}$ is the parallel wave number, $\mathbf{b}=\mathbf{B} / B$ is the field versor, and $v_{\mathrm{A}}^{2}=B^{2} /\left(\mu_{0} \rho\right)$ is the squared Alfvén speed. Near any $x^{\prime}$, the eigenvalue $\omega^{2}\left(x^{\prime}\right)=k_{\|}^{2}\left(x^{\prime}\right) v_{\mathrm{A}}^{2}\left(x^{\prime}\right)$ defines a singular solution $\tilde{\xi}_{x} \propto K_{0}\left(k\left|x-x^{\prime}\right|\right)$, with $K_{0}(x)=-\ln x+\cdots$ the modified Bessel function of the second kind [5]. Unlike the discrete spectra of Sturm-Liouville operators (whose coefficients do not vanish in their domain), Eq. (3) produces a set of eigenvalues $\omega(x)$ that depend continuously on the variable $x$ along the inhomogenity direction (i.e., a continuum). Likewise, eigenvalues in the continuum of the more general Eq. (1) define singular waves that travel along field lines at $v_{\mathrm{A}}$ (transverse $\mathbf{b} \times \boldsymbol{\xi} \times \mathbf{b}$ or shear-Alfvén waves) or at the sound speed (parallel $\boldsymbol{\xi} \cdot \mathbf{b}$ or acoustic waves), the square of the latter being $c_{\mathrm{S}}^{2}=\gamma P / \rho[1-$ 3]. Regardless of their polarisation, singular continuum waves are known to be strongly damped $[6,7]$.
On tokamaks, $k_{\|}=0$ at rational surfaces while $v_{\mathrm{A}}$ grows unbounded as $\rho \rightarrow 0$ at the edge, and continuum frequencies should thus span the range $0 \leqslant \omega<\infty[2,8]$. However, the field $\mathbf{B}(\Psi, \vartheta)$ depends on a poloidal angle $\vartheta$ (besides $2 \pi \Psi$, the poloidal-field flux labelling magnetic surfaces) and the consequent periodicity of the refractive index opens frequency gaps in the continua (i.e., forbidden bands) [9-11] where traveling singular waves are replaced by non-singular discrete Alfvén eigenmodes (AEs). Streaming along field lines at speeds close to $v_{\mathrm{A}}$, fusion-born $\alpha$-particles or other energetic ions produced by the heating systems may resonantly lose energy to these potentially less damped AEs [12-14], leaving the plasma core in the process. Such AE-induced transport of very hot near-Alfvénic particles (i.e., with $v \sim v_{\mathrm{A}}$ ) may hinder the operation of future fusion reactors (burn quench, wall damage, etc.) [15] and, being so, research on ion-AE interactions [16-18] have focused mostly on gaps in the shear-Alfvén (SA) continuum, all near or above the frequency $\omega_{\mathrm{TAE}}=v_{\mathrm{A}} /\left(2 q R_{0}\right)$ of toroidicity-induced AEs (TAEs), with $q \sim 1$ the safety factor and $R_{0}$ the torus major radius $[10,19,20]$.

Experimental evidences of unstable AEs with frequencies $\omega \lesssim \omega_{\text {TAE }}$ have raised the interest for gaps in the acoustic continuum also, particularly when the plasma beta $\beta \sim c_{\mathrm{S}}^{2} / v_{\mathrm{A}}^{2}$ lies in the range $2 \% \lesssim \beta \lesssim 4 \%$ [21-25]. Frequency gaps below $\omega_{\text {TAE }}$ were found in numerically computed continua, at first using the slow-sound limit $\omega \gg c_{\mathrm{S}} / R_{0}[22,26]$ and then the full set of linear MHD equations [24] to describe the SA-acoustic coupling, their width being proportional to $\beta$ in either case. Inside such gaps, $\beta$-induced AEs (BAEs) were also computed $[22,24]$, their squared frequency scaling with $c_{\mathrm{S}}^{2}$ and thus with the plasma temperature [24]. Further numerical simulations $[27,28]$ found that the coupling between SA and acoustic continua, as well as the corresponding frequency gaps and AEs (therein termed Alfvén-Slow eigenmodes or ASEs) is a robust and ubiquitous feature of tokamak plasmas, being present for a large variety of $\beta$ values, $q$ profiles (monotonic, reversed, or weakly sheared), and equilibrium shaping. 
Unlike gaps in the SA continuum, for which analytical insight about the two-wave coupling and the resulting $\mathrm{AE}$ location and frequency is readily available [10], acousticwave couplings are far more complex. Initial analytical estimates for gap frequencies and loci were limited to cylindrical equilibria and decoupled continua [24], being thus of modest accuracy and practical utility. Keeping circular magnetic surfaces but allowing finite toroidicity was later shown to couple SA and acoustic waves near a rational surface [29-31], opening a gap below the sound frequency $c_{\mathrm{S}} / R_{0}$ and lifting the $\mathrm{SA}$ continuum bottom from zero up to the value

$$
\omega_{\mathrm{GAM}}=\left(c_{\mathrm{S}} / R_{0}\right) \sqrt{2+1 / q^{2}},
$$

i.e., the typical frequency of geodesic acoustic modes [32]. Additional gaps, all below the sound frequency, were found recently by further analytical developments with the same circular equilibrium model [27]. In summary, despite long-standing numerical evidences [24, 27, 28], no gaps have thus far been predicted analytically between the sound frequency and $\omega_{\mathrm{TAE}}$ other than the bottom of the uplifted SA continuum. All previous theories [27, 2931] predict gap frequencies below the former, which is much lower than the latter because $c_{\mathrm{S}}^{2} / v_{\mathrm{A}}^{2} \ll 1$. AEs at such low frequencies are expected to be strongly damped by resonant thermal ions and thus less likely to be driven unstable. Also, the aforementioned coupling models are unable to fully explain measurements of Alfvénic activity (frequency and radial position) recently reported to have been observed in JET experiments, with frequencies lying precisely between $\omega_{\mathrm{GAM}}$ and $\omega_{\mathrm{TAE}}$ [33].

In this work, high-order plasma shaping (elongation and shift) is shown to couple SA and acoustic continua, opening frequency gaps in the range $\omega_{\mathrm{GAM}}<\omega \lesssim \omega_{\mathrm{TAE}}$. The predicted gaps lie well above the sound frequency and are a generalisation to shaped equilibria of previous analytical results [27, 29-31], all of which were obtained in the low-order limit of circular magnetic surfaces and finite toroidicity. These earlier results are briefly reviewed in Sec. II in order to grasp the need for more accurate equilibria. An equilibrium model with plasma shaping [34] is introduced and then employed to understand how each of its shaping harmonics contributes to couple SA and acoustic waves, eventually showing that shift and plasma elongation alone play a significant role in tokamaks. Insight into the coupling mechanism is developed in Sec. III, where the coupled MHD equations are expanded in powers of two small parameters: the inverse aspect ratio and the size of the shaping harmonics. This approach allows tractable equations for the coupled continua to be solved and produces analytical estimates of gap frequencies and loci, as well as an existence condition that depends on local equilibrium geometry and $q$ values. Such analytical estimates provide useful tools to interpret eventual experimental observations, as well as to develop MHD-spectroscopy techniques [35, 36]. In Sec. IV, global AEs in these gaps are found unstable to hot ions below $1 \mathrm{MeV}$ for typical tokamak parameters.
Fundamental resonances with circulating ions are shown to take place near $v^{2} \sim c_{\mathrm{S}} v_{\mathrm{A}}$. Hence, these AEs avoid strong thermal-ion damping while tapping enough energy from hot ions or fusion products. Their still unexplored role in the stability of next-step fusion experiments like ITER [37] is briefly discussed in Sec. V.

\section{CONTINUA COUPLING: EQUILIBRIA AND GEODESIC CURVATURE}

As detailed elsewhere [10, 38, 39], Eq. (1) is more conveniently written as a matrix problem whose singular continuum is the set of non-trivial solutions of the system

$$
\left[\begin{array}{cc}
\frac{\omega^{2}}{v_{\mathrm{A}}^{2}}+\frac{B}{g} \nabla_{\|}\left(\frac{g}{B} \nabla_{\|}\right) & \frac{c_{\mathrm{S}}^{2}}{v_{\mathrm{A}}^{2}} \frac{B^{2}}{g} \mathcal{K} \\
\mathcal{K} & 1+\frac{c_{\mathrm{S}}^{2}}{v_{\mathrm{A}}^{2}}+\frac{c_{\mathrm{S}}^{2}}{\omega^{2}} B \nabla_{\|}\left(\frac{1}{B} \nabla_{\|}\right)
\end{array}\right]\left[\begin{array}{l}
\xi^{\mathrm{A}} \\
\xi^{\mathrm{S}}
\end{array}\right]=0,
$$

coupling the shear-Alfvén $\xi^{\mathrm{A}}=\boldsymbol{\xi} \cdot \mathbf{B} \times \nabla \Psi / g$ and acoustic $\xi^{\mathrm{S}}=\nabla \cdot \boldsymbol{\xi}$ components of the plasma displacement $\boldsymbol{\xi}$, with $g=|\nabla \Psi|^{2}, \nabla_{\|}=\mathbf{b} \cdot \nabla$, while $\mathcal{K}=2 \boldsymbol{\kappa} \cdot \mathbf{b} \times \nabla \Psi / B$ and $\kappa=\nabla_{\|} \mathbf{b}$ are, respectively, the geodesic and field-line curvatures.

In the cylindrical-equilibrium limit, $\Psi$ and $B$ do not depend on the angle $\vartheta, \boldsymbol{\kappa}$ is parallel to $\nabla \Psi$, and the field lines are geodesics, whence $\mathcal{K} \rightarrow 0, \nabla_{\|} \rightarrow i k_{\|}$, and two decoupled continua arise from Eq. (5) as [2, 3, 8]

$$
\omega^{2}=k_{\|}^{2} v_{\mathrm{A}}^{2} \quad \text { and } \quad \omega^{2}=k_{\|}^{2} c_{\mathrm{S}}^{2} /\left(1+c_{\mathrm{S}}^{2} / v_{\mathrm{A}}^{2}\right) .
$$

In general, however, $B$ and $\Psi$ depend on $\vartheta$, the harmonics in $\xi^{\mathrm{A}}=e^{i n \phi} \sum_{m} \xi_{m}^{\mathrm{A}}(\Psi) e^{i m \vartheta}$ (and similarly for $\xi^{\mathrm{S}}$, with $\phi$ the toroidal angle around the torus) become coupled, and Eq. (5) turns into a nondiagonal algebraic system. There, each $p$-index harmonic of the periodic $\mathcal{K}(\Psi, \vartheta)$ couples in the same equation $\xi_{m}^{\mathrm{A}}$ and the pair $\xi_{m \pm p}^{\mathrm{S}}$ for integer $p$.

Low- $\beta$, high aspect-ratio equilibria with finite toroidicity and circular magnetic surfaces have $\mathcal{K} \propto \sin \vartheta$ at lowest order, being thus able to couple the three harmonics $\xi_{m}^{\mathrm{A}}$ and $\xi_{m \pm 1}^{\mathrm{S}}$ near a rational surface [29]. If the acousticwave term $\propto c_{\mathrm{S}}^{2} /\left(R_{0}^{2} \omega^{2}\right)$ in Eq. (5) is dropped under the slow-sound approximation, a SA wave $\xi^{\mathrm{A}}$ couples with the acoustic response $\xi^{\mathrm{S}}=-\mathcal{K} \xi^{\mathrm{A}} /(1+\beta)$ to its propagation and the frequency at the rational surface is lifted away from zero $[22,26]$. Keeping the acoustic-wave term, and thus the two harmonics $\xi_{m \pm 1}^{\mathrm{S}}$, brings the bottom of the lifted SA continuum slightly down to $\omega_{\mathrm{GAM}}$ and opens a gap at the beta-induced acoustic AE (BAAE) frequency $[30,31]$

$$
\omega_{\mathrm{BAAE}}=c_{\mathrm{S}} /\left(q R_{0}\right)<\omega_{\mathrm{GAM}} .
$$

The same toroidicity couples $\xi_{m}^{\mathrm{S}}$ and $\xi_{m \pm 1}^{\mathrm{S}}$ also, via the field magnitude in the term $c_{\mathrm{S}}^{2} / v_{\mathrm{A}}^{2}$, yielding additional gaps below $\omega_{\mathrm{BAAE}}[27]$. Further couplings are not possible unless higher-order harmonics are considered in $\mathcal{K}(\Psi, \vartheta)$. In the following, plasma shaping is shown to provide such harmonics, opening additional gaps above the frequency $\omega_{\mathrm{GAM}}$. 
An analytically tractable equilibrium model is built by providing a local description of the poloidal flux [34]

$$
\Psi(r, \theta)=\Psi_{\mathrm{b}} S_{0} r^{2}\left[\Theta_{0}(\theta)+\varepsilon r \Theta_{1}(\theta)+\varepsilon^{2} r^{2} \Theta_{2}(\theta)\right]
$$

depending on geometric coefficients $\left(S_{0}, \hat{\kappa}, \check{\kappa}, \hat{\Delta}, \hat{\eta}, \check{\eta}, \hat{\chi}\right.$, and $\check{\chi}$, all constant on each magnetic surface) via

$$
\begin{aligned}
\Theta_{0}(\theta)= & 1+\hat{\kappa} \cos 2 \theta+\check{\kappa} \sin 2 \theta, \\
\Theta_{1}(\theta)= & \hat{\Delta} \cos \theta+\frac{1}{4} \check{\kappa} \sin \theta+\hat{\eta} \cos 3 \theta+\check{\eta} \sin 3 \theta, \\
\Theta_{2}(\theta)= & \frac{1}{32}(8 \hat{\Delta}-3 \hat{\kappa}-3) \\
& +\frac{1}{8}(2 \hat{\eta}+2 \hat{\Delta}-\hat{\kappa}-1) \cos 2 \theta \\
& +\frac{1}{16}(4 \check{\eta}-\check{\kappa}) \sin 2 \theta+\hat{\chi} \cos 4 \theta+\check{\chi} \sin 4 \theta .
\end{aligned}
$$

Above, $r$ and $\theta$ are such that $R=R_{0}(1+\varepsilon r \cos \theta)$ is the distance to the torus axis, with $\varepsilon=a / R_{0}, a$ the minor radius, and $\Psi_{\mathrm{b}}$ the boundary flux. The field follows from $\mathbf{B}=\nabla \phi \times \nabla \Psi+B_{\phi} \nabla \phi$, with $B_{\phi}=B_{0} R_{0} \sqrt{1+\varepsilon^{2} S_{\mathrm{d}} \Psi / \Psi_{\mathrm{b}}}$ the covariant toroidal field, $B_{0}$ the field on axis, and $S_{\mathrm{d}}$ the diamagnetic coefficient. An example is illustrated in Fig. 1 for parameters typical of optimised scenarios at the Joint European Torus (JET) [40]: The equilibrium is computed by HELENA [41] and the local flux (8) is fitted to each magnetic surface to get the geometric coefficients. These change little along $\rho_{\text {pol }}=\sqrt{\Psi / \Psi_{\mathrm{b}}}$ and follow the orderings

$$
S_{0}, \hat{\Delta} \sim 1, \quad \hat{\kappa} \sim \varepsilon, \quad \text { and } \quad \check{\kappa}, \hat{\eta}, \check{\eta}, \hat{\chi}, \check{\chi} \lesssim \varepsilon^{2} .
$$

Analytical magnetic surfaces are found inverting $\Psi(r, \theta)$ for a given flux value, yielding the series [34]

$$
r(\theta)=\tilde{s}\left(\frac{1}{\Theta_{0}^{1 / 2}}-\frac{\Theta_{1}}{2 \Theta_{0}^{2}} \tilde{\varepsilon}+\frac{5 \Theta_{1}^{2}-4 \Theta_{0} \Theta_{2}}{8 \Theta_{0}^{7 / 2}} \tilde{\varepsilon}^{2}+\cdots\right),
$$

with $\tilde{s}^{2}=S_{0}^{-1}\left(\Psi / \Psi_{\mathrm{b}}\right)$ and $\tilde{\varepsilon}=\varepsilon \tilde{s}$.

Intricate functions of $\mathbf{B}$ and $\Psi$, as is the case of $\mathcal{K}$, are expanded in powers of the small numbers $\tilde{\varepsilon}$ and $\tilde{\delta} \sim \tilde{\varepsilon}$, the latter introduced here to enforce the ordering in Eqs. (10) by letting $\hat{\kappa} \rightarrow \tilde{\delta} \hat{\kappa}, \check{\kappa} \rightarrow \tilde{\delta}^{2} \check{\kappa}$, and so forth. After tracking the order of each expanded term, $\tilde{\delta}$ is replaced by 1 to restore physical formulae. Casting the real-valued $\mathcal{K}$ as

$$
\mathcal{K}(\tilde{s}, \theta)=\frac{\tilde{\varepsilon}}{\tilde{q}}\left[\mathcal{K}_{0}(\tilde{s})+\sum_{p=1}^{\infty} \mathcal{K}_{p}^{*}(\tilde{s}) e^{-i p \theta}+\mathcal{K}_{p}(\tilde{s}) e^{i p \theta}\right],
$$

where $\tilde{q}=\frac{1}{2} S_{0}^{-1} a^{2} B_{0} / \Psi_{\mathrm{b}}$ is the cylindrical $q$ at lowest order, the most important coefficients $\mathcal{K}_{p}$ (with $\mathcal{K}_{p}^{*}$ their conjugates) are

$$
\begin{aligned}
i \mathcal{K}_{1}= & 1-\frac{3}{4} \tilde{\delta} \hat{\kappa}-\frac{1}{16} \tilde{\delta}^{2}\left(\hat{\kappa}^{2}-12 i \check{\kappa}\right) \\
& -\tilde{\varepsilon}^{2}\left(\frac{1+\hat{\Delta}}{\tilde{q}^{2}}-\frac{1+4 \hat{\Delta}-6 \hat{\Delta}^{2}}{64}+\frac{1}{2} S_{0} S_{\mathrm{d}}\right)+\cdots, \\
i \mathcal{K}_{2}= & \frac{1}{4} \tilde{\varepsilon} \hat{\Delta}+\frac{1}{4} \tilde{\varepsilon} \tilde{\delta}\left(\hat{\Delta}-4 / \tilde{q}^{2}\right) \hat{\kappa}+\cdots, \\
i \mathcal{K}_{3}= & -\frac{1}{4} \tilde{\delta} \hat{\kappa}-\tilde{\varepsilon}^{2} \frac{1-2 \hat{\Delta}+3 \hat{\Delta}^{2}}{32}+\tilde{\delta}^{2} \frac{5 \hat{\kappa}^{2}+8 i \check{\kappa}}{32}+\cdots, \\
i \mathcal{K}_{5}= & \frac{3}{32} \tilde{\delta}^{2} \hat{\kappa}^{2}+\cdots,
\end{aligned}
$$
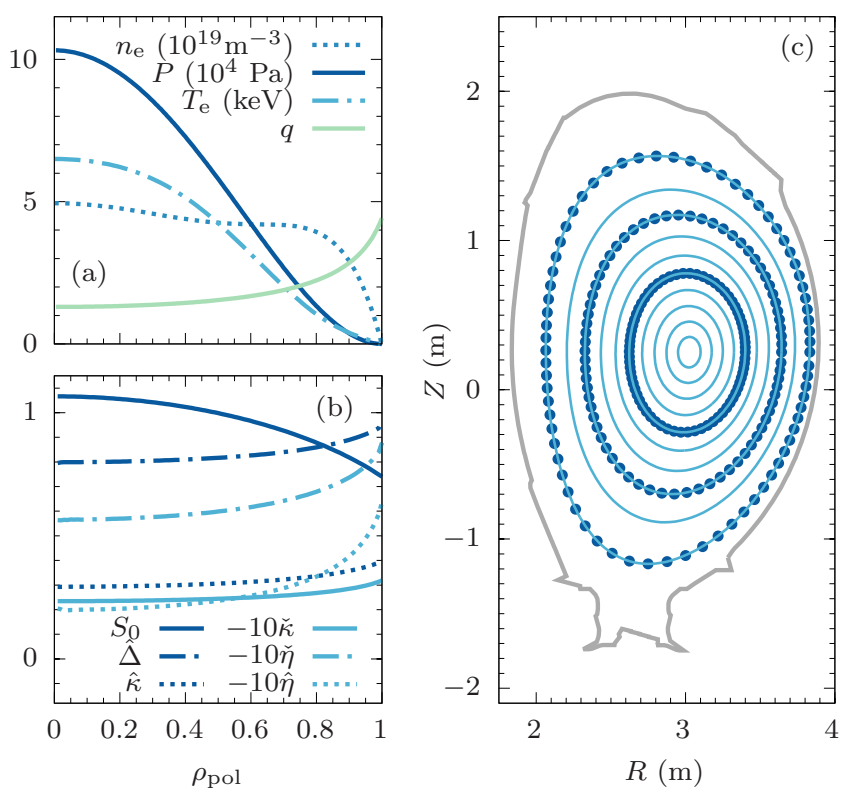

FIG. 1. JET-like equilibrium with $\varepsilon=0.29, R_{0}=3 \mathrm{~m}$, $B_{0}=3.4 \mathrm{~T}$, and $I_{\mathrm{p}}=2.3 \mathrm{MA}$ : (a) Electron density $n_{\mathrm{e}}$ and temperature $T_{\mathrm{e}}$, plasma pressure $P$, and safety factor $q$; (b) fitted coefficients $S_{0}, \hat{\Delta}, \hat{\kappa}, \check{\kappa}, \hat{\eta}$, and $\check{\eta}$; (c) numerical (solid lines) and analytical (large dots) magnetic surfaces.

all others being ordered as $O\left(\tilde{\varepsilon}^{3}, \tilde{\delta}^{3}\right)$. A crucial step to obtain the coefficients in Eq. (13), is the transformation $\mathcal{K}(r, \theta) \rightarrow \mathcal{K}(\tilde{s}, \theta)$ to the surface-induced coordinate set $\{\tilde{s}, \theta, \phi\}$, which is achieved thanks to the series in Eq. (11).

At lowest order, with circular magnetic surfaces and toroidicity alone, one finds $\mathcal{K}=(2 \tilde{\varepsilon} / \tilde{q}) \sin \theta+\cdots$ in agreement with earlier results $[27,29,30]$. In turn, equilibrium shaping brings in first-order corrections to the coefficients $\mathcal{K}_{p}$ listed in Eq. (13) due to finite $\hat{\Delta}$ and $\hat{\kappa}$ (for $1 \leqslant p \leqslant 3$ only), which are related with the Shafranov shift and plasma elongation [34]. The linear term in $\mathcal{K}_{1}$ changes slightly the already known coupling between $\xi_{m}^{\mathrm{A}}$ and the two harmonics $\xi_{m \pm 1}^{\mathrm{S}}$. On the other hand, those in $\mathcal{K}_{2}$ and $\mathcal{K}_{3}$ introduce additional couplings with $\xi_{m \pm 2}^{\mathrm{S}}$ and $\xi_{m \pm 3}^{\mathrm{S}}$ that may open new frequency gaps. For $p \geqslant 4$, all terms in $\mathcal{K}_{p}$ are quadratic or higher powers of $\tilde{\varepsilon}$ or $\tilde{\delta}$ and the couplings they induce are weaker, being thus discarded.

\section{FREQUENCY GAPS: ANALYTICAL ESTIMATES AND NUMERICAL VERIFICATION}

Near rational surfaces where the parallel wave-number vanishes, i.e.

$$
k_{\|} R_{0}=m / \tilde{q}+n=0,
$$

only the branch $\xi_{m}^{\mathrm{A}}$ of the SA continuum is close in frequency to the acoustic branches $\xi_{m+p}^{\mathrm{S}}$ with $|p|=0,1,2,3$. Using the analytical model described in Eqs. (8) to (11) 
to deal with equilibrium quantities and differential operators, the system in Eq. (5) can be expanded in powers of the small parameters $\tilde{\varepsilon}$ and $\tilde{\delta}$. Keeping only terms up to the first order, the matrix problem is reduced to

$$
\left[\begin{array}{cccccccc}
\tilde{\rho} \tilde{\omega}^{2}-\zeta^{2} & \mathcal{K}_{3} & \mathcal{K}_{2} & \mathcal{K}_{1} & \mathcal{K}_{0} & \mathcal{K}_{1}^{*} & \mathcal{K}_{2}^{*} & \mathcal{K}_{3}^{*} \\
\mathcal{K}_{3}^{*} & D_{-3} & w_{-3}^{+} & u_{-3}^{+} & & & & \\
\mathcal{K}_{2}^{*} & w_{-2}^{-} & D_{-2} & w_{-2}^{+} & u_{-2}^{+} & & \mathbf{0} & \\
\mathcal{K}_{1}^{*} & u_{-1}^{-} & w_{-1}^{-} & D_{-1} & w_{-1}^{+} & u_{-1}^{+} & & \\
\mathcal{K}_{0} & & u_{0}^{-} & w_{0}^{-} & D_{0} & w_{0}^{+} & u_{0}^{+} & \\
\mathcal{K}_{1} & & & u_{1}^{-} & w_{1}^{-} & D_{1} & w_{1}^{+} & u_{1}^{+} \\
\mathcal{K}_{2} & & \mathbf{0} & & u_{2}^{-} & w_{2}^{-} & D_{2} & w_{2}^{+} \\
\mathcal{K}_{3} & & & & & u_{3}^{-} & w_{3}^{-} & D_{3}
\end{array}\right]\left[\begin{array}{c}
\tilde{\xi}_{m}^{\mathrm{A}} \\
\tilde{\xi}_{m-3}^{\mathrm{S}} \\
\tilde{\xi}_{m}^{\mathrm{S}} \\
\tilde{\xi}_{m-2}^{\mathrm{S}} \\
\tilde{\xi}_{m}^{\mathrm{S}} \\
\tilde{\xi}_{m}^{\mathrm{S}} \\
\tilde{\xi}_{m+1}^{\mathrm{S}} \\
\tilde{\xi}_{m+3}^{\mathrm{S}}
\end{array}\right]=0 .
$$

Here, mass density and frequency are normalised to their on-axis values as $\rho=\rho_{0} \tilde{\rho}$ and $\omega=\left(v_{\mathrm{A}}^{0} / R_{0}\right) \tilde{\omega}$, whereas the variables $\tilde{\xi}^{\mathrm{A}}=(\tilde{\varepsilon} / \tilde{q}) \xi^{\mathrm{A}}$ and $\tilde{\xi}^{\mathrm{S}}=\tilde{\beta} \xi^{\mathrm{S}}$ are thus defined in order to simplify the coefficients in Eq. (15) and to follow the ordering $\tilde{\xi}^{\mathrm{S}} \sim \tilde{\beta} \tilde{\xi}^{\mathrm{A}}$, with $\tilde{\beta}=\gamma \mu_{0} P / B_{0}^{2}$. Moreover, the principal and side diagonals are defined as

$$
\begin{aligned}
\tilde{\beta} D_{p}= & 1+\tilde{\beta}-\frac{1}{\tilde{\rho}}\left(\frac{\tilde{\beta}}{\tilde{\omega}^{2}}\right)\left(\zeta+\frac{p}{\tilde{q}}\right)^{2}, \\
\tilde{\beta} w_{p}^{ \pm}= & \tilde{\varepsilon} \tilde{\beta}-\frac{\tilde{\varepsilon}}{\tilde{\rho}}\left(\frac{\tilde{\beta}}{\tilde{\omega}^{2}}\right)[ \\
& \frac{m p}{\tilde{q}^{2}}+\frac{3}{2} \hat{\Delta} \frac{m+p \pm 1}{\tilde{q}}\left(\zeta+\frac{p \pm 1 / 2}{\tilde{q}}\right) \\
& \left.-\frac{1}{2 \tilde{q}}\left(\frac{1}{\tilde{q}} \pm \zeta\right)-\left(\zeta \pm \frac{1}{2 \tilde{q}}\right)\left(\zeta+\frac{m+p}{\tilde{q}}\right)\right], \\
\tilde{\beta} u_{p}^{ \pm}= & -\tilde{\delta}\left(\frac{\tilde{\beta}}{\tilde{\omega}^{2}}\right) \hat{\kappa} \frac{m+p \pm 2}{\tilde{q} \tilde{\rho}}\left(\zeta+\frac{p \pm 1}{\tilde{q}}\right),
\end{aligned}
$$

where $\zeta(\tilde{q})=m / \tilde{q}+n$ is the dimensionless value $R_{0} k_{\|}(\tilde{q})$ at the radial location with safety factor $\tilde{q}$. Each acoustic harmonic $\tilde{\xi}_{p}^{\mathrm{S}}$ is coupled to $\tilde{\xi}_{p \pm 1}^{\mathrm{S}}$ and $\tilde{\xi}_{p \pm 2}^{\mathrm{S}}$ by the diagonals $w_{p}^{ \pm}$and $u_{p}^{ \pm}$that arise due to toroidicity and shift and due to elongation, respectively. On the other hand, all acoustic harmonics are coupled with $\tilde{\xi}_{m}^{\mathrm{A}}$ by the geodesiccurvature coefficients that are placed along the matrix first line and column.

The ordering of terms in Eq. (15) is not exclusively set by toroidal bending and plasma shaping via the small parameters $\tilde{\varepsilon}$ and $\tilde{\delta}$. The frequency range of interest also plays a role and different continua are found if the slowsound approximation $\tilde{\beta} / \tilde{\omega}^{2} \sim 0$ is considered $[22,26]$ or if the acoustic-frequency limit $\tilde{\beta} / \tilde{\omega}^{2} \sim 1$ is taken $[30,31]$. Conversely, the focus in this work is placed on frequency values below but close to $\omega_{\mathrm{TAE}}$, which are thus ordered as $\tilde{\beta} / \tilde{\omega}^{2} \sim \tilde{\varepsilon}$ and lie between the two previous limits. Recalling that $\tilde{\xi}^{\mathrm{S}} \sim \tilde{\beta} \tilde{\xi}^{\mathrm{A}}$, all terms arising from the first line in Eq. (15) become consistently ordered if $\zeta^{2} \lesssim \tilde{\beta} / \tilde{\varepsilon}$ and, consequently, one is sufficiently close to a rational surface. In turn, the side diagonals $w_{p}^{ \pm}$and $u_{p}^{ \pm}$produce, respec- tively, terms ordered as $O\left(\tilde{\varepsilon} \tilde{\beta}, \tilde{\varepsilon}^{2}\right)$ and $O(\tilde{\varepsilon} \tilde{\delta})$ or smaller, which may be discarded when compared with those in $\mathcal{K}_{p}$ or the in main diagonal $D_{p}$. Overall, for frequencies in the range $\tilde{\beta} / \tilde{\omega}^{2} \sim \tilde{\varepsilon}$, Eq. (15) simplifies to

$$
\left[\begin{array}{ccccccc}
\tilde{\rho} \tilde{\omega}^{2}-\zeta^{2} & \mathcal{K}_{3} & \mathcal{K}_{2} & \mathcal{K}_{1} & \mathcal{K}_{1}^{*} & \mathcal{K}_{2}^{*} & \mathcal{K}_{3}^{*} \\
\mathcal{K}_{3}^{*} & D_{-3} & & & & & \\
\mathcal{K}_{2}^{*} & & D_{-2} & & & \mathbf{0} & \\
\mathcal{K}_{1}^{*} & & & D_{-1} & & & \\
\mathcal{K}_{1} & & & & D_{1} & & \\
\mathcal{K}_{2} & & \mathbf{0} & & & D_{2} & \\
\mathcal{K}_{3} & & & & & & D_{3}
\end{array}\right]\left[\begin{array}{c}
\tilde{\xi}_{m}^{\mathrm{A}} \\
\tilde{\xi}_{m}^{\mathrm{S}} \\
\tilde{\xi}_{m}^{\mathrm{S}} \mathrm{s}-2 \\
\tilde{\xi}_{m}^{\mathrm{S}} \\
\tilde{\xi}_{m}^{\mathrm{S}} \\
\tilde{\xi}_{m+1}^{\mathrm{S}} \\
\tilde{\xi}_{m+2}^{\mathrm{S}} \\
\mathrm{m}_{m+3}
\end{array}\right]=0 .
$$

Above, the line and column $p=0$ are omitted because $\mathcal{K}_{0}$ terms are $O\left(\tilde{\varepsilon} \tilde{\delta}^{2}\right)$, as noticed in Eqs. (13). Hence, $D_{0}$ factors out from the matrix determinant and $\tilde{\xi}_{m}^{\mathrm{S}}$ keeps a cylindrical continuum as in Eq. (6). In addition, one should remark that if the frequency had been ordered as $\tilde{\beta} / \tilde{\omega}^{2} \sim 1$, the side diagonals would keep coupling $\tilde{\xi}_{m}^{\mathrm{S}}$ to the other harmonics, eventually leading to frequency gaps near $\tilde{\omega}^{2} \approx \tilde{\beta} /(2 \tilde{q})^{2}$ (i.e., $\omega^{2} \approx \tilde{\beta} \omega_{\text {TAE }}^{2}$ ) as obtained elsewhere [27].

Letting $C$ be the coupling matrix in Eq. (17), continua are found solving $\operatorname{det} C\left(\rho_{\text {pol }}, \tilde{\omega}^{2}\right)=0$, which factorises as

$$
\begin{aligned}
& \tilde{\omega}^{2} \Upsilon_{1} \Upsilon_{2} \Upsilon_{3}\left(\frac{1}{2 \tilde{q}^{2}} \Upsilon_{1} \Upsilon_{2} \Upsilon_{3}\right. \\
& \left.\quad-\left|\mathcal{K}_{1}\right|^{2} \Upsilon_{2} \Upsilon_{3}-\Upsilon_{1}\left|\mathcal{K}_{2}\right|^{2} \Upsilon_{3}-\Upsilon_{1} \Upsilon_{2}\left|\mathcal{K}_{3}\right|^{2}\right)=0
\end{aligned}
$$

at a given rational surface $\zeta\left(\tilde{q}_{\ddagger}\right)=0$ labelled by the safety factor $\tilde{q}_{\ddagger}=-m / n$, with $\Upsilon_{p}=\left(\tilde{\omega} / \tilde{\omega}_{\mathrm{S}}\right)^{2}-p^{2}$ and

$$
\tilde{\omega}_{\mathrm{S}}^{2}=\frac{\tilde{\beta}}{\tilde{\rho} \tilde{q}_{\ddagger}^{2}(1+\tilde{\beta})} .
$$

Aside from the trivial solution $\tilde{\omega}=0$, the roots $\Upsilon_{p}=0$ for $p=1,2,3$ are the top branches of the three gaps located where the cylindrical continua of $\tilde{\xi}_{m \pm p}^{\mathrm{S}}$ would cross each other (Fig. 2). At integer multiples of $\tilde{\omega}_{\mathrm{S}}$, these roots are independent of the equilibrium shaping and the first one 

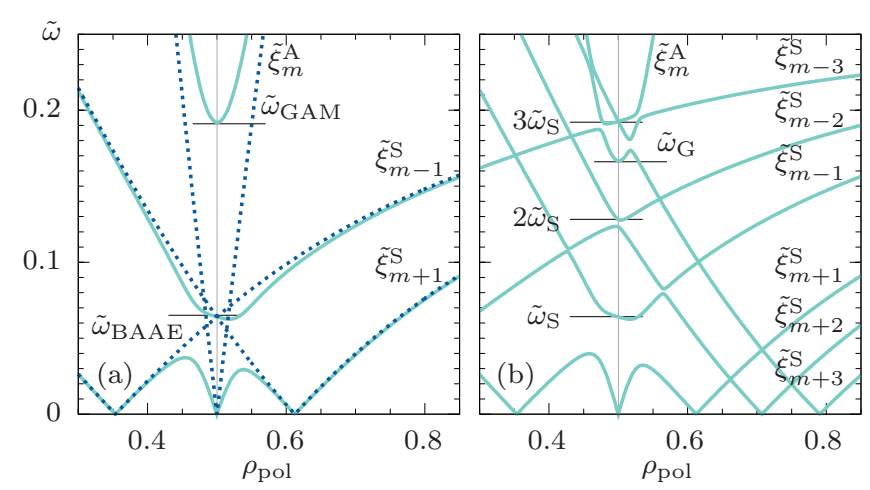

FIG. 2. Continua for $\tilde{q}\left(\rho_{\text {pol }}\right)=1+4 \rho_{\text {pol }}^{2}, \tilde{\rho}=1, \tilde{\beta}=\frac{5}{3} \frac{1}{100}$, $\varepsilon=0.3, S_{0}=1, m=4$, and $n=-2$ : (a) limit $\mathcal{K}=0$ (dots) and lowest-order coupling for circular equilibria $(\hat{\Delta}, \hat{\kappa}=0$, lines); (b) high-order couplings with $\hat{\Delta}=5$ and $\hat{\kappa}=\frac{1}{4}$.

corresponds to the well-known frequency $\omega_{\mathrm{BAAE}}$ [29-31]. In turn, the factor in brackets yields three more roots: the lower branches of the $p=2,3$ gaps at

$$
\tilde{\omega}^{2} / \tilde{\omega}_{\mathrm{S}}^{2}=p^{2}-\left(p^{2}-1\right) C_{p}\left|\mathcal{K}_{p}\right|_{2}^{2}+\cdots
$$

and the bottom of the SA continuum that is uplifted to the geodesic frequency $\tilde{\omega}_{\mathrm{G}}$ defined as

$$
\frac{\tilde{\omega}_{\mathrm{G}}^{2}}{\tilde{\omega}_{\mathrm{S}}^{2}}=1+2 \tilde{q}_{\ddagger}^{2}\left(1+\left|\mathcal{K}_{1}\right|_{1}^{2}+\sum_{p=1}^{3} C_{p}\left|\mathcal{K}_{p}\right|_{2}^{2}+\cdots\right),
$$

with $1 / C_{p}=1-\left(p^{2}-1\right) /\left(2 \tilde{q}_{\ddagger}^{2}\right)$, whereas $\left|\mathcal{K}_{1}\right|_{1}^{2}=-\frac{3}{2} \tilde{\delta} \hat{\kappa}$ and $\left|\mathcal{K}_{p}\right|_{2}^{2}$ are the linear and quadratic terms of $\left|\mathcal{K}_{p}\right|^{2}$, all of which depend on the shaping as follows from Eqs. (13). In the limit of circular equilibria $\left|\mathcal{K}_{1}\right|_{1}^{2}$ and $\left|\mathcal{K}_{p}\right|_{2}^{2}$ vanish, thus reducing the frequency $\left(v_{\mathrm{A}}^{0} / R_{0}\right) \tilde{\omega}_{\mathrm{G}}$ to $\omega_{\mathrm{GAM}}$ as obtained in previous works $[27,29-31]$ and actually closing the $p=2,3$ gaps. Because their width is $\tilde{\beta}^{\frac{1}{2}} O\left(\tilde{\varepsilon}^{2} \hat{\Delta}^{2}, \tilde{\delta}^{2} \hat{\kappa}^{2}\right)$, such gaps are only relevant for large values of $\tilde{\beta}, \hat{\Delta}$, or $\hat{\kappa}$.

Slightly away from the rational surface $\zeta\left(\tilde{q}_{\ddagger}\right)=0$, wider gaps of size $\tilde{\beta}^{\frac{1}{2}} O(\tilde{\varepsilon} \hat{\Delta}, \tilde{\delta} \hat{\kappa})$ arise if the $\tilde{\xi}_{m \pm p}^{\mathrm{S}}$ continuum crosses the one from $\tilde{\xi}_{m}^{\mathrm{A}}$ (Fig. 2), whose bottom is uplifted to $\tilde{\omega}_{\mathrm{G}}$. The condition enabling such crossings is therefore

$$
\tilde{\omega}_{\mathrm{G}}^{2}<p^{2} \tilde{\omega}_{\mathrm{S}}^{2} \quad \Leftrightarrow \quad \tilde{q}_{\ddagger}^{2}<\frac{1}{2} \frac{p^{2}-1}{1-\frac{3}{2} \tilde{\delta} \hat{\kappa}}+\cdots
$$

and gaps with $|p|=2,3$ may open if $\tilde{q}_{\ddagger} \lesssim \sqrt{3 / 2}$ or 2 , respectively, with elongation shifting these limits slightly upwards. The locus $\zeta_{p}$ of such gaps is found replacing $\tilde{\omega}^{2}=\tilde{\omega}_{S}^{2} \tilde{q}_{\ddagger}^{2}(\zeta+p / \tilde{q})^{2}$ from Eq. (6) and $\tilde{q}=\tilde{q}_{\ddagger} /(1-\zeta / n)$ into the submatrix $\mathrm{C}_{ \pm 1}\left(\tilde{\omega}^{2}, \zeta\right)$ obtained from $\mathrm{C}\left(\tilde{\omega}^{2}, \zeta\right)$ by keeping only the harmonics $\tilde{\xi}_{m}^{\mathrm{A}}$ and $\tilde{\xi}_{m \pm 1}^{\mathrm{S}}$ in Eq. (17). The condition $\operatorname{det} C_{ \pm 1}(\zeta)=0$ is solved assuming $\zeta$ to be a series in the small parameter $\varsigma=\tilde{\beta} /\left[\tilde{q}_{\ddagger}^{2}\left(1-\tilde{\omega}_{\mathrm{S}}^{2} / \tilde{\omega}_{\mathrm{G}}^{2}\right)\right]$, yielding at length for each $p$

$$
\zeta_{p}= \pm \varsigma^{\frac{1}{2}} \sqrt{p^{2}-\tilde{\omega}_{\mathrm{G}}^{2} / \tilde{\omega}_{\mathrm{S}}^{2}}+\varsigma\left(1+n p \tilde{q}_{\ddagger}-p^{2}\right) / n+\cdots .
$$

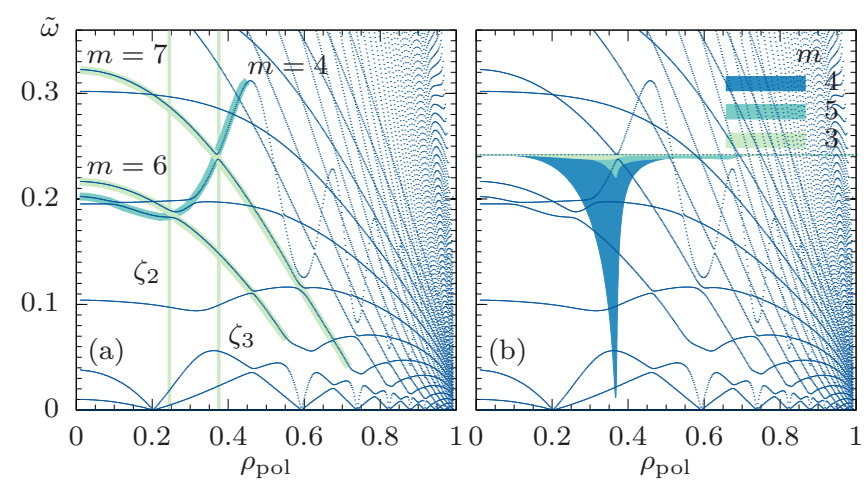

FIG. 3. Continua for the JET-like equilibrium and $n=-3$ : (a) coupling of the $\tilde{\xi}_{4}^{\mathrm{A}}, \tilde{\xi}_{6}^{\mathrm{S}}$, and $\tilde{\xi}_{7}^{\mathrm{S}}$ branches $(p=2,3)$, their gaps, and locations by $\zeta_{p} ;$ (b) global eigenmode $\left(q B_{\phi}^{-1} \boldsymbol{\xi} \cdot \nabla \rho_{\text {pol }}^{2}\right.$ in a.u., dominant harmonics only) in the $p=3$ gap.

Requiring a real-valued $\zeta_{p}$ above recovers Eq. (22), while the condition $n \tilde{q}+m=\tilde{q} \zeta_{p}$ becomes the analog of the well known rule $n \tilde{q}+m=p / 2$ that is valid for shape-induced couplings of SA continua [20].

Continua for tokamak equilibria keep the key features discussed above. The numerical results of a continuousspectrum extension [42] to the MHD code CASTOR [43] are plotted in Fig. 3, for the JET-like equilibrium of Fig. 1. Using data from the latter, one finds the values $\tilde{\beta} \approx 0.017$ and $\tilde{\omega}_{\mathrm{S}} \approx 0.1$ over the rational surface $\tilde{q}_{\ddagger}=4 / 3$ located at $\rho_{\text {pol }}=0.2$, while keeping quadratic terms in Eq. (21) yields $\tilde{\omega}_{\mathrm{G}} \approx 0.18$. All these values agree with the plotted results. Besides the tiny gaps at $2 \tilde{\omega}_{\mathrm{S}}$ and $3 \tilde{\omega}_{\mathrm{S}}$, the local value of the ratio $\tilde{\omega}_{\mathrm{G}}^{2} / \tilde{\omega}_{\mathrm{S}}^{2} \approx 3.24$ in Eq. (22) predicts the wider $p=2,3$ gaps, which are clearly visible in Fig. 3 slightly away from the rational surface. Their locations are provided by Eq. (23), after the estimates $\zeta_{2}$ and $\zeta_{3}$ are translated into safety-factor values and then converted to radial positions using the profile $q\left(\rho_{\text {pol }}\right)$ in Fig. 1. These locations correspond to the vertical lines in Fig. 3 (a), again in agreement with the plotted numerical spectrum.

Inside these high-order frequency gaps (i.e., $|p|=2,3$ due to equilibrium shaping as opposed to $|p|=1$ caused by low-order toroidicity), traveling waves are replaced by high-order geodesic-acoustic eigenmodes (HOGAEs), as the one computed by CASTOR and depicted in Fig. 3 (b). Replacing Eq. (23) in the acoustic continuum of Eq. (6), their frequency is

$$
\tilde{\omega}_{p} / \tilde{\omega}_{\mathrm{S}}=|p| \pm \varsigma^{\frac{1}{2}} \tilde{q}_{\ddagger}(1+p / m) \sqrt{p^{2}-\tilde{\omega}_{\mathrm{G}}^{2} / \tilde{\omega}_{\mathrm{S}}^{2}}+\cdots
$$

and the estimate $\tilde{\omega}_{3} \approx 0.235$ agrees well with the plotted value. Recalling that $\tilde{\omega}_{\mathrm{TAE}}=1 /(2 \tilde{q})$, one finds at lowest order from Eq. $(24)$ the ratio $\tilde{\omega}_{p} / \tilde{\omega}_{\text {TAE }} \approx 2 p \tilde{\beta}^{\frac{1}{2}} \sim p / 5$, if $\tilde{\beta} \sim 10^{-2}$ as is usually the case in tokamaks. HOGAEs with $|p|=3$ are of particular interest because, at the same location, their frequency $\tilde{\omega}_{3} \sim \frac{3}{5} \tilde{\omega}_{\mathrm{TAE}}$ is the one closest to the TAE gap. In fact, the value $\tilde{\omega}_{3}$ evaluated at 
TABLE I. Normalised growth rate due to ICRH accelerated $\mathrm{H}$ ions assuming a particle-number ratio $N_{\mathrm{H}} / N_{\mathrm{D}}=\frac{1}{100}$.

\begin{tabular}{r|rrrr}
\hline \hline$T_{\mathrm{H}}(\mathrm{keV})$ & 100 & 200 & 400 & 800 \\
\hline$\gamma_{\mathrm{H}} / \omega$ & 0.004 & 0.012 & 0.049 & 0.089 \\
\hline \hline
\end{tabular}

the plasma core can be larger than $\tilde{\omega}_{\mathrm{TAE}}$ for outer TAEs located at higher $q$. Therefore, HOGAEs may play a role similar to that of TAEs in the stability of fusion plasmas.

\section{RESONANT INTERACTIONS AND LINEAR STABILITY}

The interaction between HOGAEs and a species $s$ can be evaluated perturbatively [44] if the current density $J_{s}$ follows the condition

$$
\frac{J_{s}}{J} \sim Z_{s} \frac{n_{s}}{n_{\mathrm{e}}}\left(\frac{m_{\mathrm{e}}}{m_{s}}\right)^{\frac{1}{2}}\left(\frac{T_{s}}{T_{\mathrm{e}}}\right)^{\frac{1}{2}} \ll 1
$$

(with $Z_{s}, m_{s}, n_{s}$, and $T_{s}$ the charge number, mass, particle density, and temperature) and if the growth rate $\gamma_{s}$ is such that

$$
\frac{\gamma_{s}}{\omega}=-\operatorname{Im} \int \frac{L_{(1)}^{*} f_{s}^{(1)}}{2 \omega^{2}} d^{3} x d^{3} v / \int \rho \boldsymbol{\xi} \cdot \boldsymbol{\xi}^{*} d^{3} x \ll 1 .
$$

Here, $L_{(1)}$ and $f_{s}^{(1)}$ are the linear response of the guidingcenter Lagrangian and equilibrium distribution function $f_{s}$ to the perturbation $\boldsymbol{\xi}$ [44]. The integrals in Eq. (26) are computed by the drift-kinetic code CASTOR-K $[45,46]$ in the space of the guiding-center constants of motion: energy $E$, toroidal momentum $P_{\phi}$, and $\Lambda=\mu B_{0} / E$, with $\mu$ the magnetic moment. The results for the interaction between the $p=3$ HOGAE in Fig. 3, thermal deuterium (D, Maxwellian distribution with $T_{\mathrm{D}}=T_{\mathrm{e}}$ as in Fig. 1) and ion-cyclotron resonance heating (ICRH) $\mathrm{H}$ ions are shown in Fig. 4, assuming a separable distribution

$$
f_{\mathrm{ICRH}}\left(\rho_{\mathrm{pol}}, E, \Lambda\right) \propto \frac{1-\rho_{\mathrm{pol}}}{\sqrt{0.015+\rho_{\mathrm{pol}}}} e^{-\frac{E}{T_{\mathrm{H}}}} e^{-\frac{(\Lambda-)^{2}}{2 \delta_{\Lambda}^{2}}},
$$

with $\delta_{\Lambda}=1 / 200$ corresponding to a Doppler broadening $2 R_{0} \delta_{\Lambda}=3 \mathrm{~cm}$ of the ICRH resonant layer. Damping on thermal ions is mainly due to passing particles $(\Lambda \lesssim 1-\varepsilon)$ and $\gamma_{\mathrm{D}} / \omega=-0.0175$. Trapped ICRH ions produce drive and damping at $\Lambda=1 \pm \delta_{\Lambda}$ respectively, where $\left|\partial f_{s} / \partial \Lambda\right|$ is highest. However, the energy transfer is larger for higher $\Lambda$, whence a net drive that increases with $T_{\mathrm{H}}$. The growth rate $\gamma_{\mathrm{H}} / \omega$ is listed in Tab. I for different $T_{\mathrm{H}}$ values and the instability threshold is thus slightly above $200 \mathrm{keV}$.

For resonant interactions between AEs and particles to take place, $\omega$ must be related with the orbit-averaged frequencies $\langle\dot{\theta}\rangle$ and $\langle\dot{\phi}\rangle$ as

$$
\omega+n\langle\dot{\phi}\rangle+(l+m)\langle\dot{\theta}\rangle=0
$$
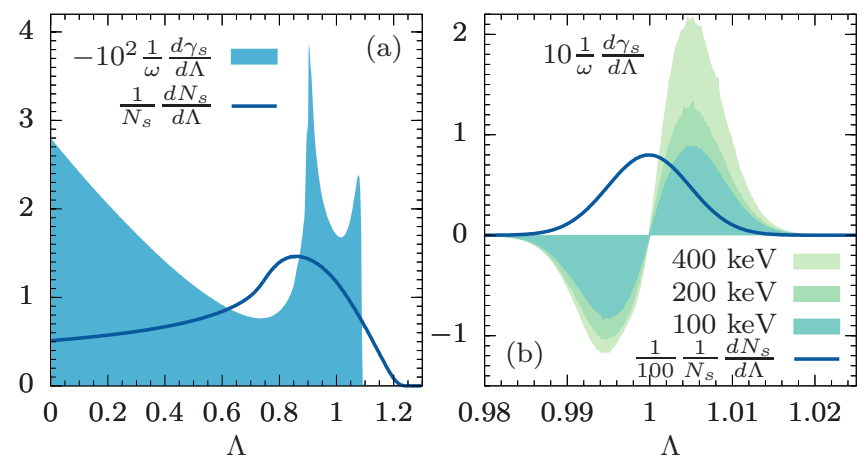

FIG. 4. Normalised linear growth rate $\gamma_{s} / \omega$ and number of particles $N_{s}$ per $\Lambda$ unit for thermal (a) and ICRH ions (b).

with $l$ an integer [20]. In the strongly passing-particle limit $\Lambda \rightarrow 0$, these are $\langle\dot{\phi}\rangle \approx q\langle\dot{\theta}\rangle \approx v_{\|} / R_{0}$ and Eq. (28) becomes $\tilde{\omega}+\left(\zeta_{p}+l / q\right)\left(v_{\|} / v_{\mathrm{A}}^{0}\right)=0$. Replacing $\tilde{\omega}$ and $\zeta_{p}$ by Eqs. (24) and (23) and solving for $v_{\|}$yields the series

$$
\frac{v_{\|}}{c_{\mathrm{S}}}=-\frac{|p|}{l \sqrt{1+\tilde{\beta}}}\left[1+\varsigma^{\frac{1}{2}} \tilde{q}_{\ddagger} C_{l, m, p} \sqrt{p^{2}-\frac{\tilde{\omega}_{\mathrm{G}}^{2}}{\tilde{\omega}_{\mathrm{S}}^{2}}}+\cdots\right],
$$

with $C_{l, m, p}= \pm(m+p) /(m|p|) \mp(l+m) /(l m)$, where the top/bottom choices for the signs correspond to those made for the gap frequency and location in Eqs. (23) and (24), respectively. The condition in Eq. (29) is the equivalent to the known relation $v_{\|} / v_{\mathrm{A}}^{0}=-p^{\prime} /(2 l+1)$ for SA eigenmodes (and, in particular, for TAEs when $\left.p^{\prime}=1\right)[20]$. The interaction of thermal and ICRH ions with the $p=3$ HOGAE in the $\left\{E, P_{\phi}\right\}$ plane is displayed in Fig. 5, with $E_{\mathrm{A}}=\frac{1}{2} m_{s} v_{\mathrm{A}}^{2}$ and $E_{\mathrm{S}}=\frac{1}{2} m_{s} c_{\mathrm{S}}^{2}$. Strongly-passing thermal ions show resonances along the gap radial location, at energy values in agreement with the estimates produced by Eq. (29) for several values of the integer number $l$. Their temperature $\left(T_{\mathrm{D}} \approx 5 \mathrm{keV}\right)$ is not sufficient to access the fundamental resonances $(|l|=1$, at $128.1 \mathrm{keV}$ and $31.2 \mathrm{keV})$ and interactions are restricted to lower sidebands $(|l| \geqslant 2)$. By their side, trapped ICRH-ion resonances depend on bounce and precession frequencies and their interaction pattern is thus more complex.

Besides the thermal-ion Landau damping discussed in the previous paragraphs, other damping mechanisms are usually taken into account when assessing the stability of AEs in fusion devices. These include the collisional damping on trapped electrons [47], the radiative damping due to finite coupling with kinetic Alfvén waves [48, 49], and the damping caused by eventual interactions with the ideal-MHD continuum [50, 51]. All these damping models, however, were developed with SA waves in mind (particularly TAEs) and, in their present form, are not suitable to handle AEs with a non-neglegible acoustic component, as is the case of HOGAEs. One noticeable exception is the evaluation of the continuum damping by taking the imaginary part of the eigenvalue $\omega+i \gamma_{\text {res }}$ of the 

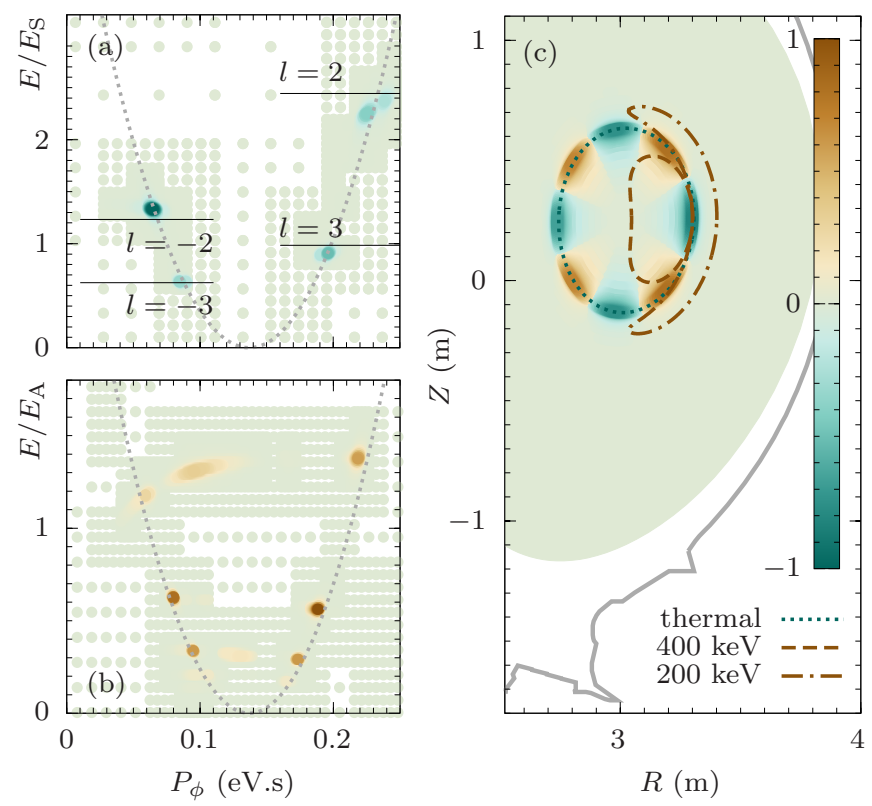

FIG. 5. Energy exchange (shading code, a.u.) due to passing $\left(\Lambda=10^{-3}\right)$ thermal (a) and trapped $\left(\Lambda=1+\delta_{\Lambda}\right)$ ICRH ions (b, $T_{\mathrm{H}}=200 \mathrm{keV}$ ), along with the gap radial location (dotted line); HOGAE poloidal structure $\left(q B_{\phi}^{-1} \boldsymbol{\xi} \cdot \nabla \rho_{\text {pol }}^{2}\right.$ in a.u. $)$ and orbits with largest energy transfer (c).

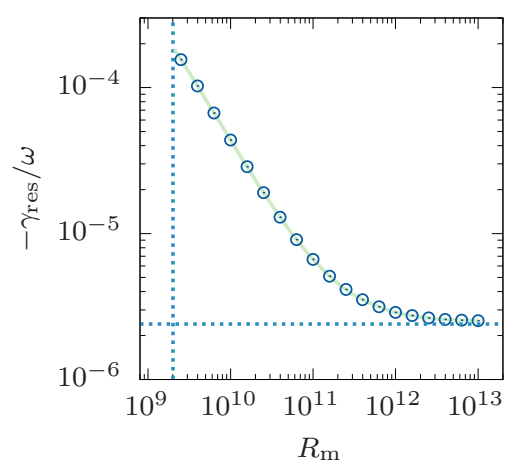

FIG. 6. Normalised damping rate $\gamma_{\mathrm{res}} / \omega$ from resistive MHD as a function of the magnetic Reynolds number $R_{\mathrm{m}}$ for the $n=-3, p=3$ HOGAE.

linear resistive-MHD problem in the limit of vanishing plasma resistivity $[52,53]$. This aim can be accomplished with the MHD code CASTOR (keeping compressibility and finite resistivity $\eta$ ) and Fig. 6 displays a scan in the magnetic Reynolds number $R_{\mathrm{m}}=\mu_{0} v_{\mathrm{A}}^{0} R_{0} / \eta$ that starts at the value of the Spitzer resistivity (corresponding to $1 / R_{\mathrm{m}} \approx 5 \times 10^{-10}$ ) and spans about four orders of magnitude. As expected, the normalised damping rate becomes independent of the resistivity for large $R_{\mathrm{m}}[52,53]$. Moreover, the asymptotic value $\gamma_{\text {res }} / \omega \approx 2.4 \times 10^{-6}$ indicates a very weak interaction between the ideal-MHD continuum and the considered HOGAE.

\section{DISCUSSION}

In summary, equilibrium shaping (mainly due to $\hat{\Delta}$ and $\hat{\kappa})$ was shown to couple acoustic and SA continua through $p$-order periodicity in $\mathcal{K}(\tilde{s}, \theta)$, leading to high-order (i.e., $|p|=2,3)$ frequency gaps at $\omega_{\mathrm{GAM}} \lesssim \omega_{p} \lesssim \omega_{\mathrm{TAE}}$ that lie significantly above the previously known toroidicityinduced BAAE gap $(|p|=1)$, if the condition in Eq. (22) is met. Inside such gaps, global HOGAEs were found to be driven unstable by anisotropic ICRH-ion populations for tokamak parameters. Circulating-ion resonances were shown to lie in the range $E \lesssim p^{2} E_{S}$, this limit being near the geometric mean $\left(E_{\mathrm{S}} E_{\mathrm{A}}\right)^{\frac{1}{2}}$ because $E_{\mathrm{S}} / E_{\mathrm{A}} \sim \tilde{\beta}$ and $p^{2} \tilde{\beta}^{\frac{1}{2}} \sim 1$ in fusion devices.

In conclusion, potentially unstable HOGAEs were found to populate the frequency range below but close to $\omega_{\mathrm{TAE}}$ in tokamak plasmas. Their location and frequency estimates in Eqs. (23) and (24) are expected to guide the interpretation of their eventual observation in experiments [33] and their role in simulations of SA-acoustic continua coupling for next-step fusion devices [54,55], as well as to foster their use in MHD spectroscopy.

Among all AEs induced by finite $\beta$ (i.e., ASEs), as those found in many numerical approaches [24, 27, 28], HOGAEs have the frequency closer to $\omega_{\mathrm{TAE}}$ and, therefore, the possibility to interact with energetic ions in a fashion similar to that of TAEs. Indeed, the resonance condition in Eq. (29) shows that HOGAEs are able to tap energy from hot ions at $E \sim \tilde{\beta}^{\frac{1}{2}} E_{\mathrm{A}}$, while avoiding efficient damping by thermal ions at the fundamental resonance $E \sim p^{2} E_{\mathrm{S}} \sim \gamma p^{2} T_{\mathrm{D}}$. Driven by less energetic ions, HOGAEs are thus expected to grow slower than TAEs that have resonant interactions at $E \sim E_{\mathrm{A}}$. Yet, they may dominate at the nonlinear saturation stage, as found numerically for low-frequency AEs close to $\omega_{\mathrm{GAM}}$ [54]. HOGAEs may hence play a significant and still unexplored role, along with TAEs, in the stability assessments of fusion reactors like ITER [56-61]. Three issues beyond the scope of this work should be pursued elsewhere: characterise HOGAEs in experimental scenarios as those recently reported [33], evaluate their interaction with isotropic $\alpha$-particles, and estimate the hot-ion redistribution and losses they may induce in burning plasmas.

\section{ACKNOWLEDGMENTS}

The authors gratefully thank Drs. S. Sharapov and M. Fitzgerald (CCFE, UK) for insightful discussions. IPFN activities were supported by Fundação para a Ciência e Tecnologia (FCT, Lisboa) via project UID/FIS/50010/2019. One of the authors (FC) was supported by FuseNet, Euratom's research and training programme in the EUROfusion Consortium, under Grant No. 633053. Views and opinions expressed here do not necessarily reflect those of the European Commission. 
[1] C. Uberoi, Phys. Fluids 15, 1673 (1972).

[2] H. Grad, Proc. Natl. Acad. Sci. USA 70, 3277 (1973).

[3] J. P. Goedbloed, Phys. Fluids 18, 1258 (1975).

[4] I. B. Bernstein, E. A. Frieman, M. D. Kruskal, and R. M. Kulsrud, Proc. Roy. Soc. Series A 244, 17 (1958).

[5] M. Abramowitz and I. A. Stegun, Handbook of Mathematical Functions, 9th ed. (Dover, 1972).

[6] J. Tataronis and W. Grossmann, Z. Physik 261, 203 (1973).

[7] W. Grossmann and J. Tataronis, Z. Physik 261, 217 (1973).

[8] K. Appert, R. Gruber, and J. Vaclavik, Phys. Fluids 17, 1471 (1974).

[9] J. W. Strutt, Phil. Mag. 24, 145 (1887).

[10] C. Z. Cheng and M. S. Chance, Phys. Fluids 29, 3695 (1986).

[11] Y. Zhang, W. W. Heidbrink, H. Boehmer, R. McWilliams, G. Chen, B. N. Breizman, S. Vincena, T. Carter, D. Leneman, W. Gekelman, P. Pribyl, and B. Brugman, Phys. Plasmas 15, 012103 (2008).

[12] M. N. Rosenbluth and P. H. Rutherford, Phys. Rev. Lett. 34, 1428 (1975).

[13] G. Y. Fu and J. W. V. Dam, Phys. Fluids B 1, 1949 (1989).

[14] R. Betti and J. P. Freidberg, Phys. Fluids B 4, 1465 (1992).

[15] A. Fasoli, C. Gormenzano, H. Berk, B. Breizman, S. Briguglio, D. Darrow, N. Gorelenkov, W. Heidbrink, A. Jaun, S. Konovalov, R. Nazikian, J.-M. Noterdaeme, S. Sharapov, K. Shinohara, D. Testa, K. Tobita, Y. Todo, G. Vlad, and F. Zonca, Nucl. Fusion 47, S264 (2007).

[16] W. Heidbrink and G. Sadler, Nucl. Fusion 34, 535 (1994).

[17] N. Gorelenkov, S. Pinches, and K. Toi, Nucl. Fusion 54, 125001 (2014).

[18] P. Lauber, Phys. Rep. 533, 33 (2013).

[19] R. Betti and J. P. Freidberg, Phys. Fluids B 3, 1865 (1991).

[20] W. W. Heidbrink, Phys. Plasmas 15, 055501 (2008).

[21] W. W. Heidbrink, E. J. Strait, M. S. Chu, and A. D. Turnbull, Phys. Rev. Lett. 71, 855 (1993).

[22] A. D. Turnbull, E. J. Strait, W. W. Heidbrink, M. S. Chu, H. H. Duong, J. M. Greene, L. L. Lao, T. S. Taylor, and S. J. Thompson, Phys. Fluids B 5, 2546 (1993).

[23] W. W. Heidbrink, E. Ruskov, E. M. Carolipio, J. Fang, M. A. van Zeeland, and R. A. James, Phys. Plasmas 6, 1147 (1999).

[24] G. T. A. Huysmans, W. Kerner, D. Borba, H. A. Holties, and J. P. Goedbloed, Phys. Plasmas 2, 1605 (1995).

[25] W. Heidbrink, M. V. Zeeland, M. Austin, N. Crocker, X. Du, G. McKee, and D. Spong, Nucl. Fusion 61, 066031 (2021).

[26] M. S. Chu, J. M. Greene, L. L. Lao, A. D. Turnbull, and M. S. Chance, Phys. Fluids B 4, 3713 (1992).

[27] C. Z. Cheng, G. J. Kramer, M. Podesta, and R. Nazikian, Phys. Plasmas 26, 082508 (2019).

[28] G. J. Kramer, C. Z. Cheng, M. Podestà, and R. Nazikian, Plasma Phys. Control. Fusion 62, 075012 (2020).

[29] B. van der Holst, A. J. C. Beliën, and J. P. Goedbloed, Phys. Plasmas 7, 4208 (2000).

[30] N. Gorelenkov, H. Berk, E. Fredrickson, S. Sharapov, and J. E. Contributors, Phys. Lett. A 370, 70 (2007).
[31] N. N. Gorelenkov, H. L. Berk, N. A. Crocker, E. D. Fredrickson, S. Kaye, S. Kubota, H. Park, W. Peebles, S. A. Sabbagh, S. E. Sharapov, D. Stutmat, K. Tritz, F. M. Levinton, and H. Y. and, Plasma Phys. Control. Fusion 49, B371 (2007).

[32] N. Winsor, J. L. Johnson, and J. M. Dawson, Phys. Fluids 11, 2448 (1968).

[33] P. Rodrigues, D. Borba, F. Cella, R. Coelho, J. Ferreira, A. Figueiredo, M. Mantsinen, F. Nabais, S. Sharapov, P. Sirén, and JET Contributors, in 28th IAEA Fusion Energy Conference (Nice, France, 10-15 May, 2021).

[34] P. Rodrigues and A. Coroado, Nucl. Fusion 58, 106040 (2018).

[35] J. P. Goedbloed, H. A. Holties, S. Poedts, G. T. A. Huysmans, and W. Kerner, Plasma Phys. Control. Fusion 35, B277 (1993).

[36] A. Fasoli, D. Testa, S. Sharapov, H. L. Berk, B. Breizman, A. Gondhalekar, R. F. Heeter, M. Mantsinen, and contributors to the EFDA-JET Workprogramme, Plasma Phys. Control. Fusion 44, B159 (2002).

[37] R. Aymar, P. Barabaschi, and Y. Shimomura, Plasma Phys. Control. Fusion 44, 519 (2002).

[38] E. Hameiri, Phys. Fluids 24, 562 (1981).

[39] E. Hameiri, Comm. Pure Appl. Math. 38, 43 (1985).

[40] R. J. Dumont, J. Mailloux, V. Aslanyan, M. Baruzzo, C. Challis, I. Coffey, A. Czarnecka, E. Delabie, J. Eriksson, J. Faustin, J. Ferreira, M. Fitzgerald, J. Garcia, L. Giacomelli, C. Giroud, N. Hawkes, P. Jacquet, E. Joffrin, T. Johnson, D. Keeling, D. King, V. Kiptily, B. Lomanowski, E. Lerche, M. Mantsinen, L. Meneses, S. Menmuir, K. McClements, S. Moradi, F. Nabais, M. Nocente, A. Patel, H. Patten, P. Puglia, R. Scannell, S. Sharapov, E. R. Solano, M. Tsalas, P. Vallejos, and H. W. and, Nucl. Fusion 58, 082005 (2018).

[41] G. Huysmans, J. Goedbloed, and W. Kerner, Int. J. Mod. Phys. C 2, 371 (1991).

[42] S. Poedts and E. Schwartz, J. Comput. Phys. 105, 165 (1993).

[43] W. Kerner, J. Goedbloed, G. Huysmans, S. Poedts, and E. Schwarz, J. Comput. Phys. 142, 271 (1998).

[44] F. Porcelli, R. Stankiewicz, W. Kerner, and H. L. Berk, Phys. Plasmas 1, 470 (1994).

[45] D. Borba and W. Kerner, J. Comput. Phys. 153, 101 (1999).

[46] F. Nabais, D. Borba, R. Coelho, A. Figueiredo, J. Ferreira, N. Loureiro, and P. Rodrigues, Plasma Sci. Technol. 17, 89 (2015).

[47] N. N. Gorelenkov and S. E. Sharapov, Phys. Scr. 45, 163 (1992).

[48] R. R. Mett and S. M. Mahajan, Phys. Fluids B 4, 2885 (1992).

[49] J. Candy and M. N. Rosenbluth, Phys. Plasmas 1, 356 (1994).

[50] M. N. Rosenbluth, H. L. Berk, J. W. Van Dam, and D. M. Lindberg, Phys. Rev. Lett. 68, 596 (1992).

[51] F. Zonca and L. Chen, Phys. Rev. Lett. 68, 592 (1992).

[52] S. Poedts and W. Kerner, Phys. Rev. Lett. 66, 2871 (1991).

[53] S. Poedts, W. Kerner, J. P. Goedbloed, B. Keegan, G. T. A. Huysmans, and E. Schwarz, Plasma Phys. Control. Fusion 34, 1397 (1992). 
[54] Y. Todo and A. Bierwage, Plasma Fusion Res. 9, 3403068 (2014).

[55] A. Bierwage, N. Aiba, and K. Shinohara, Phys. Rev. Lett. 114, 015002 (2015).

[56] S. D. Pinches, I. T. Chapman, P. W. Lauber, H. J. C. Oliver, S. E. Sharapov, K. Shinohara, and K. Tani, Phys. Plasmas 22, 021807 (2015).

[57] P. Lauber, Plasma Phys. Control. Fusion 57, 054011 (2015).

[58] P. Rodrigues, A. Figueiredo, J. Ferreira, R. Coelho,
F. Nabais, D. Borba, N. Loureiro, H. Oliver, and S. Sharapov, Nucl. Fusion 55, 083003 (2015).

[59] A. Figueiredo, P. Rodrigues, D. Borba, R. Coelho, L. Fazendeiro, J. Ferreira, N. Loureiro, F. Nabais, S. Pinches, A. Polevoi, and S. Sharapov, Nucl. Fusion 56, 076007 (2016).

[60] M. Fitzgerald, S. Sharapov, P. Rodrigues, and D. Borba, Nucl. Fusion 56, 112010 (2016).

[61] M. Schneller, P. Lauber, and S. Briguglio, Plasma Phys. Control. Fusion 58, 014019 (2016). 